\title{
Linear forms in two logarithms and interpolation determinants
}

by

\author{
Michel Laurent (Marseille)
}

1. Introduction. Our aim is to test numerically the new method of interpolation determinants (cf. [2], [6]) in the context of linear forms in two logarithms. In the recent years, M. Mignotte and M. Waldschmidt have used Schneider's construction in a series of papers [3]-[5] to get lower bounds for such a linear form with rational integer coefficients. They got relatively precise results with a numerical constant around a few hundreds. Here we take up Schneider's method again in the framework of interpolation determinants. We decrease the constant to less than one hundred when the logarithms involved are real numbers. Theorems 1 and 2 are simple corollaries of our main result which is Theorem 3. At first glance, the statement of Theorem 3 seems to be complicated, but it is much more precise than the above mentioned corollaries, which are only examples of applications. Let us also mention that we have been led in Section 3 to some technical lemmas which may be useful in some other situations apart from transcendental number theory.

A preliminary version of this text can also be found in [6], in the form of an appendix to lectures given by M. Waldschmidt at Madras Math. Science Institute. I would like to thank Dong Ping Ping and M. Waldschmidt for useful comments and remarks during the writing of this paper.

2. Statement of the results. Let $\alpha_{1}$ and $\alpha_{2}$ be two real algebraic numbers which are supposed to be $\geq 1$ and multiplicatively independent. We shall give lower bounds for the linear form

$$
\Lambda=b_{2} \log \alpha_{2}-b_{1} \log \alpha_{1},
$$

where $b_{1}$ and $b_{2}$ are rational integers which can be supposed to be $\geq 1$ without loss of generality.

For any algebraic number $\alpha$ of degree $d$ over $\mathbb{Q}$ and whose minimal polynomial over $\mathbb{Z}$ is written as $a \prod_{i=1}^{d}\left(X-\alpha^{(i)}\right)$ where the roots $\alpha^{(i)}$ are 
complex numbers, let us denote by

$$
h(\alpha)=\frac{1}{d}\left(\log |a|+\sum_{i=1}^{d} \log \max \left(1,\left|\alpha^{(i)}\right|\right)\right)
$$

the usual (Weil's) absolute logarithmic height of $\alpha$.

Let $D$ be the degree over $\mathbb{Q}$ of the number field $\mathbb{Q}\left(\alpha_{1}, \alpha_{2}\right)$, and let $a_{1}, a_{2}$ be two real numbers $>1$ such that

$$
h\left(\alpha_{i}\right) \leq \log a_{i} \quad(i=1,2) .
$$

For each pair of integers $b_{1} \geq 1, b_{2} \geq 1$, set

$$
b^{\prime}=\frac{b_{1}}{D \log a_{2}}+\frac{b_{2}}{D \log a_{1}} .
$$

Our first result gives the asymptotical value of the constant $c$ when $b^{\prime}$ tends to infinity.

TheOREM 1. For each number $c>48$, there exists a number $b^{\prime}(c)$ such that

$$
\log |\Lambda| \geq-c D^{4}\left(\log b^{\prime}\right)^{2} \log a_{1} \log a_{2}
$$

for each pair of integers $b_{1} \geq 1, b_{2} \geq 1$ with $b^{\prime} \geq b^{\prime}(c)$.

We can of course compute effectively such a constant $b^{\prime}(c)$ in term of $c$. Here is a concrete example.

Theorem 2. Suppose that $\log a_{1} \geq 1, \log a_{2} \geq 1$ and $\log b^{\prime} \geq 25$. Then

$$
\log |\Lambda| \geq-87 D^{4}\left(0.5+\log b^{\prime}\right)^{2} \log a_{1} \log a_{2} .
$$

Our main result is the following

Theorem 3. Let $K$ be an integer $\geq 2$, let $L, R_{1}, R_{2}, S_{1}, S_{2}$ be integers $\geq 1$ and let $\varrho$ be a real number $\geq 1$. Suppose that

$$
R_{1} S_{1} \geq \max (K, L), \quad R_{2} S_{2} \geq 2 K L .
$$

Define

$$
\begin{gathered}
R=R_{1}+R_{2}-1, \quad S=S_{1}+S_{2}-1, \quad \gamma=R S /(K L), \\
g=\frac{1}{4}-\frac{1}{12 \gamma}+\max \left(\frac{1}{4 \gamma L^{2}}, \frac{\gamma}{4 L R^{2}}, \frac{\gamma}{4 L S^{2}}\right) .
\end{gathered}
$$

For integers $b_{1} \geq 1, b_{2} \geq 1$, set

$$
b=\left((R-1) b_{2}+(S-1) b_{1}\right)\left(\prod_{k=1}^{K-1} k !\right)^{-2 /\left(K^{2}-K\right)} .
$$

Suppose now that $\alpha_{1}$ and $\alpha_{2}$ are multiplicatively independent, that the numbers $r b_{2}+s b_{1}(0 \leq r \leq R-1,0 \leq s \leq S-1)$ are pairwise distinct, 
and that

$$
\begin{aligned}
& K(L-1) \log \varrho+(K-3) \log 2 \\
& >2 D \log (K L)+D(K-1) \log b \\
& \quad+g L\left((\varrho-1)\left(R \log \alpha_{1}+S \log \alpha_{2}\right)+2 D\left(R \log a_{1}+S \log a_{2}\right)\right) .
\end{aligned}
$$

Then we have the lower bound

$$
\left|\Lambda^{\prime}\right| \geq \varrho^{-K L+1 / 2}
$$

where

$$
\Lambda^{\prime}=\Lambda \max \left(\frac{L S e^{L S|\Lambda| /\left(2 b_{2}\right)}}{2 b_{2}}, \frac{L R e^{L R|\Lambda| /\left(2 b_{1}\right)}}{2 b_{1}}\right) .
$$

Theorems 1 and 2 will be deduced from Theorem 3 by plugging the inequalities $\alpha_{i} \leq a_{i}^{D}(i=1,2)$ in condition (2) for specific values of the parameters $K, L, R_{1}, R_{2}, S_{1}, S_{2}$ and $\varrho$.

3. Technical lemmas. We shall have to investigate the determinant of a matrix whose entries are monomials in $\alpha_{1}$ and $\alpha_{2}$. It is crucial to know what sort of monomials appear in the expansion of this determinant. To that purpose, we shall use some combinatorial results which have been gathered in this section because their statements are independent of the original problem.

Lemma 1. Let $K, S$ and $N$ be integers $\geq 1$. Then

$\sum_{\nu=1}^{N}\left(\left[\frac{\nu-1}{K}\right]+1\right)\left(\left[\frac{N-\nu}{S}\right]+1\right) \geq \frac{N\left(2 N^{2}+3 K N+3 S N+3 K S+1\right)}{12 K S}$.

Proof. Denote the left hand side by $E$. We shall decompose $E$ into subsums corresponding to the congruence classes of $\nu$ modulo $K$ and $S$ successively.

If $\nu$ is congruent to $k$ modulo $K$, where $1 \leq k \leq K$, then $[(\nu-1) / K]=$ $(\nu-k) / K$, so that we can write

$$
E=\frac{1}{K}\left(\sum_{\nu=1}^{N} \nu a_{\nu}\right)+\frac{1}{K}\left(\sum_{k=1}^{K}(K-k) S_{k}\right),
$$

where we have set

$$
a_{\nu}=\left[\frac{N-\nu}{S}\right]+1, \quad S_{k}=\sum_{\nu} a_{\nu},
$$

and where $\nu$ in $S_{k}$ is congruent to $k$ modulo $K$.

Note that the sequence $\left(a_{\nu}\right)_{1 \leq \nu \leq N}$ is non-increasing, so that the sequence $\left(S_{k}\right)_{1 \leq k \leq K}$ of partial sums is also non-increasing. By Abel's summation, we get 


$$
\sum_{k=1}^{K}(K-k) S_{k}=\sum_{k=1}^{K-1} \sum_{j=1}^{k} S_{j} \geq \sum_{k=1}^{K-1} k S_{k}
$$

from which it follows that

$$
\sum_{k=1}^{K}(K-k) S_{k} \geq \frac{K}{2} \sum_{k=1}^{K-1} S_{k} \geq \frac{K-1}{2} \sum_{k=1}^{K} S_{k},
$$

the last term being equal to $((K-1) / 2)\left(\sum_{\nu=1}^{N} a_{\nu}\right)$. In this first step, we have got the lower bound

$$
E \geq \frac{1}{K} \sum_{\nu=1}^{N}\left(\nu+\frac{K-1}{2}\right)\left(\left[\frac{N-\nu}{S}\right]+1\right)=\frac{1}{K} \sum_{\nu=1}^{N}\left(\left[\frac{\nu-1}{S}\right]+1\right) b_{\nu},
$$

where we have set $b_{\nu}=N-\nu+((K+1) / 2)(1 \leq \nu \leq N)$. The sequence $\left(b_{\nu}\right)_{1 \leq \nu \leq N}$ is also non-increasing. The same argument, with $K$ replaced by $S$ and $\left(a_{\nu}\right)$ replaced by $\left(b_{\nu}\right)$, provides the lower bound

$$
\sum_{\nu=1}^{N}\left(\left[\frac{\nu-1}{S}\right]+1\right) b_{\nu} \geq \frac{1}{S} \sum_{\nu=1}^{N}\left(\nu+\frac{S-1}{2}\right) b_{\nu},
$$

from which it follows that

$$
E \geq \frac{1}{K S} \sum_{\nu=1}^{N}\left(\nu+\frac{S-1}{2}\right)\left(N-\nu+1+\frac{K-1}{2}\right) .
$$

But the last sum is elementarily seen to be equal to

$$
N\left(2 N^{2}+3 K N+3 S N+3 K S+1\right) / 12,
$$

and the lemma is proven.

The next lemma is also computational.

Lemma 2. Let $N$ and $S$ be natural integers. Then

$$
\sum_{\nu=1}^{N}\left[\frac{\nu-1}{S}\right] \leq \frac{(2 N-S)^{2}}{8 S}
$$

Pr o of. Denote the left hand side by $F$. If $N \leq S$, then $F=0$ while the right hand side is $\geq 0$. Suppose now that $N>S$. By Euclidean division, we can write $N=(a+1) S+b(1 \leq b \leq S, a \geq 0)$. Then

$$
\begin{aligned}
F & =\sum_{\nu=1}^{(a+1) S}\left[\frac{\nu-1}{S}\right]+\sum_{\nu=(a+1) S+1}^{N}(a+1)=\frac{S\left(a^{2}+a\right)}{2}+(a+1) b \\
& =\frac{N^{2}-S N+b(S-b)}{2 S} \leq \frac{N^{2}}{2 S}-\frac{N}{2}+\frac{S}{8}
\end{aligned}
$$

because $b$ is located between 1 and $S$. 
Let $K$ and $L$ be integers $\geq 1$, and let $N=K L$. Define

$$
l_{\nu}=\left[\frac{\nu-1}{K}\right] \quad(1 \leq \nu \leq N) \text {, }
$$

so that $\left(l_{\nu}\right)_{1 \leq \nu \leq N}$ is nothing but the sequence of integers $(0, \ldots, L-1)$, repeated $K$ times and arranged in increasing order. The next lemma will be directly used to estimate our determinants.

LEMMA 3. Under the above notation, let furthermore $R$ and $S$ be integers $\geq 1$. For each sequence $\left(r_{1}, \ldots, r_{N}\right)$ of integers between 0 and $R-1$, with any given integer repeated at most $S$ times, we have the estimate

$$
M-G \leq \sum_{\nu=1}^{N} l_{\nu} r_{\nu} \leq M+G
$$

where

$$
\begin{gathered}
M=\frac{(L-1)\left(r_{1}+\ldots+r_{N}\right)}{2}, \quad G=\frac{N L R}{2}\left(\frac{1}{4}-\frac{1}{12 \gamma}+\varepsilon\right), \\
\gamma=\frac{R S}{K L}, \quad \varepsilon=\max \left(\frac{1}{4 \gamma L^{2}}, \frac{\gamma}{4 L R^{2}}\right) .
\end{gathered}
$$

Proof. In other words, the problem is to estimate the oscillation of the sum

$$
\sigma=\sum_{\nu=1}^{N}\left(l_{\nu}-\frac{L-1}{2}\right) r_{\nu}
$$

when $\left(r_{1}, \ldots, r_{N}\right)$ runs over the set of sequences of $N$ integers, with values between 0 and $R-1$, in which a given integer appears at most $S$ times. Note first that the terms of $\sigma$ with $1 \leq \nu \leq(N+1) / 2$ are $\leq 0$, while those with $(N+1) / 2 \leq \nu \leq N$ are $\geq 0$. The symmetry

$$
l_{N-\nu+1}+l_{\nu}=L-1 \quad(1 \leq \nu \leq N)
$$

allows us to write $\sigma$ in the form

$$
\sigma=-\sum_{(N+1) / 2 \leq \nu \leq N}\left(l_{\nu}-\frac{L-1}{2}\right) r_{N-\nu+1}+\sum_{(N+1) / 2 \leq \nu \leq N}\left(l_{\nu}-\frac{L-1}{2}\right) r_{\nu} \text {. }
$$

To estimate of the above sums, we have to distinguish two cases, according to the parity of $L$.

(i) Suppose that $L$ is odd. Define $N^{\prime}=K(L-1) / 2$. In this case,

$$
\sum_{(N+1) / 2 \leq \nu \leq N}\left(l_{\nu}-\frac{L-1}{2}\right) r_{\nu}=\sum_{\nu=1}^{N^{\prime}}\left(\left[\frac{\nu-1}{K}\right]+1\right) r_{N^{\prime}+K+\nu}
$$


and $\sigma$ is the difference of two numbers of the form

$$
\beta=\sum_{\nu=1}^{N^{\prime}}\left(\left[\frac{\nu-1}{K}\right]+1\right) b_{\nu}
$$

where $\left(b_{1}, \ldots, b_{N^{\prime}}\right)$ denotes a sequence of $N^{\prime}$ integers between 0 and $R-1$ with each value appearing at most $S$ times. It follows that

$$
-\max \beta+\min \beta \leq \sigma \leq \max \beta-\min \beta .
$$

The substitution $b_{\nu} \mapsto R-1-b_{\nu}$ shows that

$$
\begin{aligned}
\max \beta+\min \beta & =(R-1) \sum_{\nu=1}^{N^{\prime}}\left(\left[\frac{\nu-1}{K}\right]+1\right) \\
& =\frac{1}{8}(R-1) K\left(L^{2}-1\right),
\end{aligned}
$$

giving the upper bound

$$
|\sigma| \leq \frac{(R-1) K\left(L^{2}-1\right)}{8}-2 \min \beta .
$$

We have to find the value $\min \beta$. Let us show that

$$
\min \beta=\sum_{\nu=1}^{N^{\prime}}\left(\left[\frac{\nu-1}{K}\right]+1\right)\left[\frac{N^{\prime}-\nu}{S}\right],
$$

that is to say that the minimal value is reached for the sequence $b_{\nu}=$ $\left[\left(N^{\prime}-\nu\right) / S\right]\left(1 \leq \nu \leq N^{\prime}\right)$. Note first that for each minimal sequence $\left(b_{\nu}\right)$, we have $b_{i} \geq b_{j}$ whenever $[(i-1) / K]<[(j-1) / K]$. Indeed, if we denote by $\left(b_{\nu}^{\prime}\right)$ the sequence obtained from $\left(b_{\nu}\right)$ by permuting $b_{i}$ and $b_{j}$, we have

$$
\sum_{\nu=1}^{N^{\prime}}\left(\left[\frac{\nu-1}{K}\right]+1\right)\left(b_{\nu}^{\prime}-b_{\nu}\right)=\left(\left[\frac{j-1}{K}\right]-\left[\frac{i-1}{K}\right]\right)\left(b_{i}-b_{j}\right),
$$

which must be $\geq 0$ by the minimal property of $\left(b_{\nu}\right)$. As the value of the sum

$$
\sum_{\nu=1}^{N^{\prime}}\left(\left[\frac{\nu-1}{K}\right]+1\right) b_{\nu}
$$

is invariant under permutations in each subsequence $\left(b_{1}, \ldots, b_{K}\right),\left(b_{K+1}, \ldots\right.$ $\left.\ldots, b_{2 K}\right), \ldots$, we may suppose without restriction that the sequence $\left(b_{\nu}\right)$ is non-increasing. By minimality, it is then clear that the $S$ last values $b_{N^{\prime}}, \ldots, b_{N^{\prime}-S+1}$ are necessarily equal to zero, the $S$ preceding ones are equal to one, and so on. In other words, we have $b_{\nu}=\left[\left(N^{\prime}-\nu\right) / S\right]$ for 
$1 \leq \nu \leq N^{\prime}$. We have proven the upper bound

$$
\begin{aligned}
|\sigma| \leq & \frac{(R-1) K\left(L^{2}-1\right)}{8} \\
& -2 \sum_{\nu=1}^{N^{\prime}}\left(\left[\frac{\nu-1}{K}\right]+1\right)\left(\left[\frac{N^{\prime}-\nu}{S}\right]+1\right)+2 \sum_{\nu=1}^{N^{\prime}}\left(\left[\frac{\nu-1}{K}\right]+1\right) .
\end{aligned}
$$

The second sum in the right hand side is equal to $K\left(L^{2}-1\right) / 8$, while Lemma 1, with $N$ replaced by $N^{\prime}$, gives the lower bound

$$
\begin{aligned}
\frac{K(L-1)}{24 K S}\left(\frac{K^{2}(L-1)^{2}}{2}+\right. & \left.\frac{3 K^{2}(L-1)}{2}+\frac{3 K S(L+1)}{2}+1\right) \\
& \geq \frac{1}{48 S}\left(K^{2}\left(L^{3}-3 L+2\right)+3 K S\left(L^{2}-1\right)\right)
\end{aligned}
$$

for the sum in the middle term. Putting all together and using the trivial estimate

we finally get

$$
\frac{(R-1) K\left(L^{2}-1\right)}{8} \leq \frac{R K L^{2}}{8}-\frac{K\left(L^{2}-1\right)}{8},
$$

$$
|\sigma| \leq \frac{R K L^{2}}{8}-\frac{K^{2} L^{3}}{24 S}+\frac{K^{2} L}{8 S}-\frac{K^{2}}{12 S}
$$

Neglecting the last term, we can write

$$
|\sigma| \leq \frac{N L R}{2}\left(\frac{1}{4}-\frac{1}{12 \gamma}+\frac{1}{4 \gamma L^{2}}\right) \leq G .
$$

(ii) Suppose now that $L$ is even. Set $N^{\prime}=K L / 2=N / 2$. In this case,

$$
\sum_{(N+1) / 2 \leq \nu \leq N}\left(l_{\nu}-\frac{L-1}{2}\right) r_{\nu}=\sum_{\nu=1}^{N^{\prime}}\left(\left[\frac{\nu-1}{K}\right]+\frac{1}{2}\right) r_{\nu+N^{\prime}} .
$$

The proof runs along the same lines, with sums of the form

$$
\beta=\sum_{\nu=1}^{N^{\prime}}\left(\left[\frac{\nu-1}{K}\right]+\frac{1}{2}\right) b_{\nu}
$$

for which, with corresponding notations, we obtain the upper bound

$$
|\sigma| \leq(R-1)\left(\sum_{\nu=1}^{N^{\prime}}\left(\left[\frac{\nu-1}{K}\right]+\frac{1}{2}\right)\right)-2 \sum_{\nu=1}^{N^{\prime}}\left(\left[\frac{\nu-1}{K}\right]+\frac{1}{2}\right)\left[\frac{N^{\prime}-\nu}{S}\right] .
$$

The right side is better written as

$$
\frac{(R+1) K L^{2}}{8}-2 \sum_{\nu=1}^{N^{\prime}}\left(\left[\frac{\nu-1}{K}\right]+1\right)\left(\left[\frac{N^{\prime}-\nu}{S}\right]+1\right)+\sum_{\nu=1}^{N^{\prime}}\left(\left[\frac{\nu-1}{S}\right]+1\right) \text {. }
$$


In the same way, using Lemmas 1 and 2, we finally get $|\sigma| \leq \frac{R K L^{2}}{8}-\frac{K^{2} L^{3}}{24 S}-\frac{L}{12 S}+\frac{S}{8}=\frac{N L R}{2}\left(\frac{1}{4}-\frac{1}{12 \gamma}-\frac{1}{6 \gamma K^{2} L^{2}}+\frac{\gamma}{4 L R^{2}}\right)$.

Neglecting the third term, we obtain $|\sigma| \leq G$.

Rem ark. The upper bound $|\sigma| \leq N L R / 8$ can be proven very easily in the following way. We write

$$
\sigma=\sum_{\nu=1}^{N}\left(l_{\nu}-\frac{L-1}{2}\right) r_{\nu}=\sum_{\nu=1}^{N}\left(l_{\nu}-\frac{L-1}{2}\right)\left(r_{\nu}-\eta\right)
$$

for each complex number $\eta$, because the average value of the sequence $\left(l_{\nu}\right)_{1 \leq \nu \leq N}$ is $(L-1) / 2$. Choosing the center $\eta=(R-1) / 2$ and bounding $\left|r_{\nu}-\eta\right| \leq(R-1) / 2$, we get

$$
|\sigma| \leq \frac{R-1}{2} \sum_{\nu=1}^{N}\left|l_{\nu}-\frac{L-1}{2}\right| .
$$

If we suppose for instance that $L$ is odd, the last sum is easily seen to be $K\left(L^{2}-1\right) / 4$. We get

$$
|\sigma| \leq \frac{K(R-1)\left(L^{2}-1\right)}{8} \leq \frac{N L R}{8},
$$

and the same for $L$ even. Lemmas 1 and 2 lead to subtracting $1 /(24 \gamma)$ from $1 / 8$.

4. Zero estimate. Let $K, L, R_{1}, R_{2}, S_{1}, S_{2}$ be integers $\geq 1$. As in Theorem 3 , put

$$
R=R_{1}+R_{2}-1, \quad S=S_{1}+S_{2}-1 .
$$

Let $b_{1}$ and $b_{2}$ be two complex numbers. For positive integers $n$ and $p$, denote as usual by $\left(\begin{array}{l}n \\ p\end{array}\right)=n \ldots(n-p+1) / p$ ! the binomial coefficient, and denote by $A$ the $K L \times R S$ matrix with entries

$$
\left(\begin{array}{c}
r b_{2}+s b_{1} \\
k
\end{array}\right) \alpha_{1}^{l r} \alpha_{2}^{l s}
$$

where $(k, l)(0 \leq k \leq K-1,0 \leq l \leq L-1)$ is the row index, while $(r, s)$ $(0 \leq r \leq R-1,0 \leq s \leq S-1)$ is the column index. It will be convenient to number the rows by setting

$$
k_{i}=i-1-K\left[\frac{i-1}{L}\right], \quad l_{i}=\left[\frac{i-1}{K}\right] \quad(1 \leq i \leq K L) .
$$

The order of the columns is irrelevant. Various zero estimates can show that under suitable conditions, the matrix $A$ is of maximal rank. Here is an example. 
Lemma 4. Suppose that

$$
R_{1} S_{1} \geq \max (K, L) \quad \text { and } \quad R_{2} S_{2} \geq 2 K L .
$$

Suppose also that the numbers $\alpha_{1}$ and $\alpha_{2}$ are multiplicatively independent and that the $R S$ numbers $r b_{2}+s b_{1}(0 \leq r \leq R-1,0 \leq s \leq S-1)$ are all distinct. Then the rank of the matrix $A$ is equal to $K L$.

P r o of. We have to show that the $K L$ rows of $A$ are linearly independent. If not, there would exist a non-zero polynomial $P[X, Y]$, with degree in $X$ bounded by $K-1$ and degree in $Y$ bounded by $L-1$, vanishing at the points

$$
\left(r b_{2}+s b_{1}, \alpha_{1}^{r} \alpha_{2}^{s}\right) \quad(0 \leq r \leq R-1,0 \leq s \leq S-1) .
$$

Now Proposition 4.1 of [4] shows that the assumptions of the lemma cannot be satisfied. Notice that our hypotheses are stronger than those of Proposition 4.1, and that the strict inequalities (a), (b), (c) in this proposition become the inequalities (1), because of a shift by one in the degrees. Of course, a suspicious reader could object that the set of points we consider is not the same as in Proposition 4.1. One can answer that, first, when $R_{1}, R_{2}, S_{1}, S_{2}$ are odd, the two sets of points differ by a translation in $\mathbb{G}_{a} \times \mathbb{G}_{m}$, and secondly, that if one of the parameters is even, the proof runs along the same lines!

5. Arithmetical lower bounds for minors of $A$. We begin the proof of Theorem 3. So we have six parameters $K, L, R_{1}, R_{2}, S_{1}, S_{2}$ satisfying (1) and integers $b_{1} \geq 1, b_{2} \geq 1$ which are almost linearly independent in the sense that the numbers $r b_{2}+s b_{1}(0 \leq r \leq R-1,0 \leq s \leq S-1)$ are pairwise distinct. By Lemma 4, the matrix $A$ associated with this set of data is of maximal $\operatorname{rank} N:=K L$. Let $\Delta$ be a non-zero $N \times N$ minor of $A$. For a suitable ordering of columns in $\Delta$, we can write

$$
\Delta=\operatorname{det}\left(\left(\begin{array}{c}
r_{j} b_{2}+s_{j} b_{1} \\
k_{i}
\end{array}\right) \alpha_{1}^{l_{i} r_{j}} \alpha_{2}^{l_{i} s_{j}}\right)_{1 \leq i, j \leq N} .
$$

The aim of this section is to prove the following lower bound for $|\Delta|$.

Lemma 5. Define

$$
\begin{gathered}
g=\frac{1}{4}-\frac{1}{12 \gamma}+\max \left(\frac{1}{4 \gamma L^{2}}, \frac{\gamma}{4 L R^{2}}, \frac{\gamma}{4 L S^{2}}\right), \\
G_{1}=g L R N / 2, \quad G_{2}=g L S N / 2, \\
M_{1}=(L-1)\left(r_{1}+\ldots+r_{N}\right) / 2, \quad M_{2}=(L-1)\left(s_{1}+\ldots+s_{N}\right) / 2 .
\end{gathered}
$$

Then

$$
\begin{aligned}
\log |\Delta| \geq & -(D-1) \log (N !)+\left(M_{1}+G_{1}\right) \log \alpha_{1}+\left(M_{2}+G_{2}\right) \log \alpha_{2} \\
& -2 D G_{1} \log a_{1}-2 D G_{2} \log a_{2}-\frac{1}{2}(D-1)(K-1) N \log b .
\end{aligned}
$$


(Recall that we have defined

$$
\left.b=\left((R-1) b_{2}+(S-1) b_{1}\right)\left(\prod_{k=1}^{K-1} k !\right)^{-2 /\left(K^{2}-K\right)} .\right)
$$

Proof. For any polynomial $P$ with integer coefficients, denote as usual by $L(P)$ the length of $P$, that is, the sum of the absolute values of the coefficients of $P$.

Let us now consider the polynomial

$$
P(X, Y)=\sum_{\sigma} \operatorname{sg}(\sigma) \prod_{i=1}^{N}\left(\begin{array}{c}
r_{\sigma(i)} b_{2}+s_{\sigma(i)} b_{1} \\
k_{i}
\end{array}\right) X^{\Sigma_{i=1}^{N} l_{i} r_{\sigma(i)}} Y^{\Sigma_{i=1}^{N} l_{i} s_{\sigma(i)}},
$$

where $\sigma$ runs over the symmetric group $\mathcal{S}_{N}$, and $\operatorname{sg}(\sigma)$ means the signature of the permutation $\sigma$. By expanding the determinant $\Delta$, we get $\Delta=P\left(\alpha_{1}, \alpha_{2}\right)$. As

$$
\left(\begin{array}{c}
r_{j} b_{2}+s_{j} b_{1} \\
k_{i}
\end{array}\right) \leq \frac{\left((R-1) b_{2}+(S-1) b_{1}\right)^{k_{i}}}{k_{i} !} \quad(1 \leq i \leq N),
$$

and $\sum_{i=1}^{N} k_{i}=(K-1) N / 2$, we easily see that $L(P)$ is bounded by

$$
\frac{N !\left((R-1) b_{2}+(S-1) b_{1}\right)^{(K-1) N / 2}}{\prod_{i=1}^{N} k_{i} !}=N ! b^{(K-1) N / 2} .
$$

To get a good lower bound for $|\Delta|$, we have to notice that $P$ is divisible by a large power of $X$ and $Y$. More precisely, Lemma 3 gives the estimates

$$
\begin{aligned}
& M_{1}-G_{1} \leq \sum l_{i} r_{\sigma(i)} \leq M_{1}+G_{1}, \\
& M_{2}-G_{2} \leq \sum l_{i} s_{\sigma(i)} \leq M_{2}+G_{2} .
\end{aligned}
$$

Denote by $V_{1}$ (resp. $V_{2}$ ) the integer part of $M_{1}+G_{1}$ (resp. $M_{2}+G_{2}$ ), and by $U_{1}$ (resp. $\left.U_{2}\right)$ the least integer $\geq M_{1}-G_{1}$ (resp. $\left.M_{2}-G_{2}\right)$. Then we can write

$$
\Delta=P\left(\alpha_{1}, \alpha_{2}\right)=\alpha_{1}^{V_{1}} \alpha_{2}^{V_{2}} \widetilde{P}\left(\frac{1}{\alpha_{1}}, \frac{1}{\alpha_{2}}\right),
$$

where $\widetilde{P}(X, Y)$ is a polynomial with integer coefficients, with the same length as $P$, and whose degree in $X$ (resp. $Y)$ is bounded by $V_{1}-U_{1}$ (resp. $\left.V_{2}-U_{2}\right)$. As $h\left(1 / \alpha_{1}\right)=h\left(\alpha_{1}\right)$ and $h\left(1 / \alpha_{2}\right)=h\left(\alpha_{2}\right)$, Liouville's inequality, in the form of Lemma 2.3 from [4], gives the lower bound

$$
\begin{aligned}
\log \left|\widetilde{P}\left(\frac{1}{\alpha_{1}}, \frac{1}{\alpha_{2}}\right)\right| \geq & -(D-1) \log L(\widetilde{P}) \\
& -D\left(V_{1}-U_{1}\right) \log a_{1}-D\left(V_{2}-U_{2}\right) \log a_{2} .
\end{aligned}
$$


Taking into account the above upper bound for $L(P)=L(\widetilde{P})$, we get

$$
\begin{aligned}
\log |\Delta| \geq & -(D-1) \log (N !)+V_{1} \log \alpha_{1}+V_{2} \log \alpha_{2} \\
& -D\left(V_{1}-U_{1}\right) \log a_{1}-D\left(V_{2}-U_{2}\right) \log a_{2} \\
& -\frac{1}{2}(D-1)(K-1) N \log b .
\end{aligned}
$$

Now, from the inequalities $D \log a_{i} \geq \log \alpha_{i} \geq 0$, we get

$$
V_{i} \log \alpha_{i}-D\left(V_{i}-U_{i}\right) \log a_{i} \geq\left(M_{i}+G_{i}\right) \log \alpha_{i}-2 D G_{i} \log a_{i}
$$

for $i=1,2$, which implies the conclusion of Lemma 5 .

6. Analytic upper bound for $|\Delta|$. Here is the crucial point where the smallness of $|\Lambda|$ is used essentially.

LEMma 6. Let $\varrho$ be a real number $\geq 1$. Suppose that

$$
\left|\Lambda^{\prime}\right| \leq \varrho^{-N+1 / 2} .
$$

Then

$$
|\Delta| \leq \varrho^{-\left(N^{2}-N\right) / 2} 2^{N}(N !)\left(\frac{\varrho b}{2}\right)^{(K-1) N / 2} \alpha_{1}^{M_{1}+\varrho G_{1}} \alpha_{2}^{M_{2}+\varrho G_{2}} .
$$

Proof. Without loss of generality, we may assume that

$$
b_{1} \log \alpha_{1} \leq b_{2} \log \alpha_{2},
$$

so that $\Lambda \geq 0$. Set $\beta=b_{1} / b_{2}$. Then

$$
\log \alpha_{2}=\beta \log \alpha_{1}+\Lambda / b_{2} .
$$

Let us first modify slightly the matrix whose $\Delta$ is the determinant. For any complex number $\eta$, as

$$
\left(\begin{array}{c}
r_{j} b_{2}+s_{j} b_{1} \\
k_{i}
\end{array}\right)=\frac{b_{2}^{k_{i}}}{k_{i} !}\left(r_{j}+s_{j} \beta-\eta\right)^{k_{i}}+\left(\text { terms of degree }<k_{i}\right)
$$

we have by multilinearity

$$
\Delta=\operatorname{det}\left(\frac{b_{2}^{k_{i}}}{k_{i} !}\left(r_{j}+s_{j} \beta-\eta\right)^{k_{i}} \alpha_{1}^{l_{i} r_{j}} \alpha_{2}^{l_{i} s_{j}}\right) .
$$

Then it is convenient to center the exponents $l_{i}$ around their average value $(L-1) / 2$. In this way we get

$$
\Delta=\alpha_{1}^{M_{1}} \alpha_{2}^{M_{2}} \operatorname{det}\left(\frac{b_{2}^{k_{i}}}{k_{i} !}\left(r_{j}+s_{j} \beta-\eta\right)^{k_{i}} \alpha_{1}^{\lambda_{i} r_{j}} \alpha_{2}^{\lambda_{i} s_{j}}\right),
$$

where $\lambda_{i}=l_{i}-(L-1) / 2(1 \leq i \leq N)$. We now write

$$
\alpha_{1}^{\lambda_{i} r_{j}} \alpha_{2}^{\lambda_{i} s_{j}}=\alpha_{1}^{\lambda_{i}\left(r_{j}+s_{j} \beta\right)} e^{\lambda_{i} s_{j} \Lambda / b_{2}}=\alpha_{1}^{\lambda_{i}\left(r_{j}+s_{j} \beta\right)}\left(1+\Lambda^{\prime} \theta_{i, j}\right)
$$


with

so that

$$
\theta_{i, j}=\frac{e^{\lambda_{i} s_{j} \Lambda / b_{2}}-1}{\Lambda^{\prime}}
$$

$$
\left|\theta_{i, j}\right| \leq \frac{2 b_{2}\left(e^{\left|\lambda_{i}\right| s_{j} \Lambda / b_{2}}-1\right)}{L S \Lambda e^{L S \Lambda /\left(2 b_{2}\right)}} \leq 1
$$

(here is the unique reason for which it is better to work with $\Lambda^{\prime}$ instead of A). Plugging this expression in the determinant $\Delta$, we get

$$
\Delta=\alpha_{1}^{M_{1}} \alpha_{2}^{M_{2}}\left(\sum_{I \subseteq\{1, \ldots, N\}}\left(\Lambda^{\prime}\right)^{N-\operatorname{Card} I} \Delta_{I}\right)
$$

where

$$
\Delta_{I}=\operatorname{det}\left(\begin{array}{ccc}
c_{i, 1} & \ldots & c_{i, N} \\
\theta_{i, 1} c_{i, 1} & \ldots & \theta_{i, N} c_{i, N}
\end{array}\right)^{\}_{i \in I}}
$$

and

$$
c_{i, j}=\frac{b_{2}^{k_{i}}}{k_{i} !}\left(r_{j}+s_{j} \beta-\eta\right)^{k_{i}} \alpha_{1}^{\lambda_{i}\left(r_{j}+s_{j} \beta\right)} .
$$

As $\sum_{i=1}^{N} \lambda_{i}=0$, one can replace in $\Delta_{I}$ the quantity $c_{i, j}$ by $c_{i, j} \alpha_{1}^{-\lambda_{i} \eta}$, so that

$$
\left.\Delta_{I}=\operatorname{det}\left(\begin{array}{ccc}
\varphi_{i}\left(z_{1}\right) & \ldots & \varphi_{i}\left(z_{N}\right) \\
\theta_{i, 1} \varphi_{i}\left(z_{1}\right) & \ldots & \theta_{i, N} \varphi_{i}\left(z_{N}\right)
\end{array}\right)\right\} i \notin I
$$

where

$$
\begin{aligned}
\varphi_{i}(z) & =\frac{b_{2}^{k_{i}}}{k_{i} !} z^{k_{i}} \alpha_{1}^{\lambda_{i} z} \quad(1 \leq i \leq N), \\
z_{j} & =r_{j}+s_{j} \beta-\eta \quad(1 \leq j \leq N) .
\end{aligned}
$$

Take now $\eta=((R-1)+\beta(S-1)) / 2$, so that

$$
\left|z_{j}\right| \leq \frac{(R-1)+\beta(S-1)}{2} \quad(1 \leq j \leq N) .
$$

We next give an upper bound for $\left|\Delta_{I}\right|$. Let us consider the entire function $\Phi_{I}$ of the complex variable $x$ defined by

$$
\Phi_{I}(x)=\operatorname{det}\left(\begin{array}{ccc}
\varphi_{i}\left(x z_{1}\right) & \ldots & \varphi_{i}\left(x z_{N}\right) \\
\theta_{i, 1} \varphi_{i}\left(x z_{1}\right) & \ldots & \theta_{i, N} \varphi_{i}\left(x z_{N}\right)
\end{array}\right)^{\}_{i \in I}}
$$

so that $\Delta_{I}=\Phi_{I}(1)$. Here is the key point of our argument.

Lemma 7. For each subset $I \subseteq\{1, \ldots, N\}$ of cardinality $\nu$, the function $\Phi_{I}$ has a zero at the origin with multiplicity $\geq\left(\nu^{2}-\nu\right) / 2$. 
Pr o of. We expand each $\varphi_{i}$ with $i \in I$ in Taylor's series at the origin:

$$
\varphi_{i}(z)=\sum_{n \geq 0} p_{i, n} z^{n} .
$$

Plugging these expressions into the determinant defining $\Phi_{I}$, we obtain

$$
\Phi_{I}(x)=\sum_{n_{i}, i \in I}\left(\prod_{i \in I} p_{i, n_{i}} x^{n_{i}}\right) \operatorname{det}\left(\begin{array}{ccc}
z_{1}^{n_{i}} & \ldots & z_{N}^{n_{i}} \\
\theta_{i, 1} \varphi_{i}\left(x z_{1}\right) & \ldots & \theta_{i, N} \varphi_{i}\left(x z_{N}\right)
\end{array}\right)^{\}_{i \in I}}
$$

where the summation indices $n_{i}, i \in I$, run a priori independently from zero to infinity. But we see that if $n_{\alpha}=n_{\beta}$ for some distinct $\alpha$ and $\beta$ in $I$, then the corresponding term in the sum is zero, because rows $\alpha$ and $\beta$ in the determinant are equal. So we may restrict our summation index to the set of $n_{i}, i \in I$, which are pairwise distinct. For such indices, we obviously have

$$
\sum_{i \in I} n_{i} \geq 0+\ldots+(\nu-1)=\frac{\nu^{2}-\nu}{2} .
$$

It follows that $\Phi_{I}$ has a zero at the origin of order $\geq\left(\nu^{2}-\nu\right) / 2$.

The usual Schwarz lemma then implies

$$
\left|\Delta_{I}\right|=\left|\Phi_{I}(1)\right| \leq \varrho^{-\left(\nu^{2}-\nu\right) / 2} \max _{|x|=\varrho}\left|\Phi_{I}(x)\right|
$$

for any real number $\varrho \geq 1$. Using these inequalities for all subsets $I \subseteq$ $\{1, \ldots, N\}$, together with

$$
\left|\Lambda^{\prime}\right| \leq \varrho^{-N+1 / 2},
$$

and substituting in $(*)$, we get

$$
|\Delta| \leq \alpha_{1}^{M_{1}} \alpha_{2}^{M_{2}} 2^{N} \max _{0 \leq \nu \leq N}\left(\varrho^{-(N-1 / 2)(N-\nu)-\left(\nu^{2}-\nu\right) / 2}\right) \max _{I} \max _{|x|=\varrho}\left|\Phi_{I}(x)\right| .
$$

As

$$
\min _{0 \leq \nu \leq N}\left(\left(N-\frac{1}{2}\right)(N-\nu)+\frac{\nu^{2}-\nu}{2}\right)=\frac{N^{2}-N}{2}
$$

we get

$$
|\Delta| \leq \alpha_{1}^{M_{1}} \alpha_{2}^{M_{2}} 2^{N} \varrho^{-\left(N^{2}-N\right) / 2} \max _{I} \max _{|x|=\varrho}\left|\Phi_{I}(x)\right| .
$$

Then Lemma 6 is an immediate consequence of the following upper bound:

Lemma 8. For each subset $I \subseteq\{1, \ldots, N\}$ and each complex number $x$, we have

$$
\left|\Phi_{I}(x)\right| \leq N !\left(\frac{|x| b}{2}\right)^{(K-1) N / 2} \alpha_{1}^{|x| G_{1}} \alpha_{2}^{|x| G_{2}}
$$


Pr o o f. Since $\left|\theta_{i, j}\right| \leq 1$, expanding the determinant $\Phi_{I}(x)$ shows that

$$
\left|\Phi_{I}(x)\right| \leq N ! \max _{\sigma}\left|\prod_{i=1}^{N} \varphi_{i}\left(x z_{\sigma(i)}\right)\right|,
$$

where $\sigma$ runs over all permutations $\sigma \in \mathcal{S}_{N}$. We have

$$
\prod_{i=1}^{N} \varphi_{i}\left(x z_{\sigma(i)}\right)=\prod_{i=1}^{N} \frac{\left(b_{2} x z_{\sigma(i)}\right)^{k_{i}}}{k_{i} !} \alpha_{1}^{\left(\Sigma \lambda_{i} z_{\sigma(i)}\right) x},
$$

and

$$
\begin{aligned}
\sum \lambda_{i} z_{\sigma(i)} & =\sum \lambda_{i}\left(r_{\sigma(i)}+\beta s_{\sigma(i)}-\eta\right) \\
& =\sum \lambda_{i}\left(r_{\sigma(i)}+\beta s_{\sigma(i)}\right)=\left(\sum \lambda_{i} r_{\sigma(i)}\right)+\beta\left(\sum \lambda_{i} s_{\sigma(i)}\right) .
\end{aligned}
$$

Now Lemma 3 gives respectively the upper bound $G_{1}$ and $G_{2}$ for the absolute values of the last two sums. We get

$$
\left|\sum \lambda_{i} z_{\sigma(i)}\right| \leq G_{1}+\beta G_{2} .
$$

By assumption $\alpha_{1}^{\beta} \leq \alpha_{2}$. Finally, the exponential term in the product $\prod_{i=1}^{N} \varphi_{i}\left(x z_{\sigma(i)}\right)$ is bounded by $\alpha_{1}^{|x| G_{1}} \alpha_{2}^{|x| G_{2}}$, as was to be shown. For the monomial term, it is enough to use the simple bound

$$
\left|z_{j}\right| \leq \frac{(R-1)+\beta(S-1)}{2} \quad(1 \leq j \leq N)
$$

so that

$$
\begin{aligned}
& \prod_{i=1}^{N} \frac{\left|b_{2} x z_{\sigma(i)}\right|^{k_{i}}}{k_{i} !} \\
& \quad \leq\left(\frac{b_{2}|x|((R-1)+\beta(S-1))}{2}\right)^{(K-1) N / 2}\left(\prod_{k=1}^{K-1} k !\right)^{-L}=\left(\frac{|x| b}{2}\right)^{(K-1) N / 2} .
\end{aligned}
$$

Remark. The determinant $\Delta$ is just the interpolation determinant of the $N$ functions in two variables $x, y$,

$$
\varphi_{i}(x, y)=\frac{b_{2}^{k_{i}}}{k_{i} !} x^{k_{i}} \alpha_{1}^{l_{i} x} e^{l_{i} y} \quad(1 \leq i \leq N),
$$

evaluated at the $N$ points

$$
\left(r_{j}+\beta s_{j}, s_{j} \Lambda / b_{2}\right) \quad(1 \leq j \leq N) .
$$

For bounding such a determinant, the general pattern is to expand it in a Taylor series (around the origin or any other point) of the $2 N$ variables $x_{j}, y_{j}(1 \leq j \leq N)$, determined by the coordinates of the given $N$ points.

In our special case, the second coordinate $y$ is small, so it has been sufficient to expand $\varphi_{i}(x, y)$ to order 1 in $y$. 
7. End of the proof of Theorem 3. On the contrary, suppose that the conditions of Theorem 3 are satisfied and that

$$
\left|\Lambda^{\prime}\right| \leq \varrho^{-K L+1 / 2} \text {. }
$$

Lemmas 5 and 6 allow us to give the following estimate for $\log |\Delta|$ :

$$
\begin{gathered}
-(D-1) \log (N !)+\left(M_{1}+G_{1}\right) \log \alpha_{1}+\left(M_{2}+G_{2}\right) \log \alpha_{2} \\
-2 D\left(G_{1} \log a_{1}+G_{2} \log a_{2}\right)-\frac{1}{2}(D-1)(K-1) N \log b \\
\leq \log |\Delta| \leq N \\
+\left(M_{1}+\varrho G_{1}\right) \log 2+\log (N !)+\frac{1}{2}(K-1) N \log (\varrho b / 2) \\
+\left(M_{2}+\varrho G_{2}\right) \log \alpha_{2} \\
-\frac{1}{2}\left(N^{2}-N\right) \log \varrho .
\end{gathered}
$$

The quantities involving $M_{1}$ and $M_{2}$ cancel on both sides of the above inequalities. We finally get the opposite of (2) by bounding $\log (N !) \leq N \log N$ and replacing $N, G_{1}, G_{2}$ by their values. This contradiction proves Theorem 3.

8. Proof of Theorems 1 and 2. As $\alpha_{1} \leq a_{1}^{D}$ and $\alpha_{2} \leq a_{2}^{D}$, it is sufficient to check instead of (2) the stronger, but simpler, inequality

$$
\begin{aligned}
& K(L-1) \log \varrho+(K-3) \log 2 \\
& \quad>2 D \log (K L)+D(K-1) \log b+g(\varrho+1) D L\left(R \log a_{1}+S \log a_{2}\right) .
\end{aligned}
$$

Now, we have to compare $b$ and $b^{\prime}$. This will be provided by

Lemma 9. For any integers $R \geq 1, S \geq 1$ and $K \geq 2$, we have

$$
b \leq \frac{5\left((R-1) b_{2}+(S-1) b_{1}\right)}{K-1} .
$$

Proof. We are led to give a uniform lower bound for

$$
\left(\prod_{k=1}^{K-1} k !\right)^{2 /\left(K^{2}-K\right)} \text {. }
$$

Let us show that this quantity is $\geq(K-1) / 5$ for any $K \geq 2$, which is the meaning of Lemma 9. This is a problem of standard calculus. One can proceed as follows. First notice that

$$
\prod_{k=1}^{K-1} k !=\prod_{k=1}^{K-1}(K-k)^{k}=\prod_{k=1}^{K-1} k^{K-k}=\frac{((K-1) !)^{K}}{\prod_{k=1}^{K-1} k^{k}} .
$$

Now we are reduced to giving an upper bound for $\prod_{k=1}^{K-1} k^{k}$. Let us show that

$$
\sum_{k=1}^{K-1} k \log k \leq \frac{K^{2}-K}{2} \log (K-1)-\frac{K^{2}-K}{4}+\frac{K}{3},
$$


for $K \geq 2$. We use Euler-Maclaurin's summation formula, in the notations of formula (7.2.4), p. 303 of [1]:

$$
f(1)+\ldots+f(n)=\int_{1}^{n} f(x) d x+\frac{f(1)+f(n)}{2}+\frac{f^{\prime}(n)-f^{\prime}(1)}{12}+R_{1}
$$

with $r=1$ and

$$
R_{1} \leq \frac{1}{2 \pi^{2}} \int_{1}^{n}\left|f^{(3)}(x)\right| d x .
$$

We take $f(x)=x \log x$ and $n=K-1$ to get

$$
\begin{aligned}
\sum_{k=1}^{K-1} k \log k \leq & \frac{(K-1)^{2}}{2} \log (K-1)-\frac{(K-1)^{2}}{4}+\frac{1}{4} \\
& +\frac{(K-1) \log (K-1)}{2}+\frac{\log (K-1)}{12}+\frac{1}{2 \pi^{2}},
\end{aligned}
$$

and it is enough to bound $\log (K-1) \leq K-2$ for any $K \geq 2$. Now, we use the standard lower bound

$$
(K-1) ! \geq(K-1)^{K-1} e^{-(K-1)} .
$$

Putting all together, we get

$$
\left(\prod_{k=1}^{K-1} k !\right)^{2 /\left(K^{2}-K\right)} \geq(K-1) e^{-3 / 2-2 /(3(K-1))} \geq \frac{K-1}{5},
$$

for $K \geq 8$. If $K=2, \ldots, 7$, the inequality between the left hand side and the right hand side is obvious.

The principle of the proofs of Theorems 1 and 2 is as follows. In each case, we shall define a system of parameters $K, L, R_{1}, R_{2}, S_{1}, S_{2}, \varrho$ satisfying the conditions (1) and (3). Theorem 3 provides a lower bound for $\left|\Lambda^{\prime}\right|$, and consequently for $|\Lambda|$, if we assume that the numbers $r b_{1}+s b_{2}(0 \leq r \leq R-1$, $0 \leq s \leq S-1)$ are pairwise distinct. If this last condition is not satisfied, Liouville's inequality furnishes a much better lower bound for $|\Lambda|$ than the one which is required.

Let $c_{1}>0, c_{2}>0, c_{3}>0, c_{4}>0$ and $f$ be constants (that is to say, numbers independent of $b^{\prime}, a_{1}$, and $a_{2}$ ) which will be defined in each case. For simplicity, define $B=f+\log b^{\prime}$. We set

$$
\begin{aligned}
K & =\left[c_{1} D^{3} B \log a_{1} \log a_{2}\right], & L & =\left[c_{2} D B\right], \\
R_{1} & =\left[c_{3} D^{3 / 2} B^{1 / 2} \log a_{2}\right]+1, & R_{2} & =\left[c_{4} D^{2} B \log a_{2}\right], \\
S_{1} & =\left[c_{3} D^{3 / 2} B^{1 / 2} \log a_{1}\right]+1, & S_{2} & =\left[c_{4} D^{2} B \log a_{1}\right] .
\end{aligned}
$$

Let us begin with Theorem 1 for which the computations are simpler because it suffices to compare the leading terms for large $B$ in the inequalities 
involved. First, thanks to Lemma 9, we bound

$$
b \leq \frac{5\left((R-1) b_{2}+(S-1) b_{1}\right)}{K-1} \leq e^{f} b^{\prime}=e^{B},
$$

if we have chosen $f>\log 5+\log c_{4}-\log c_{1}$. To satisfy (1) and (3) for large $B$, it is enough to choose positive constants $c_{1}, \ldots, c_{4}$ so that

$$
\begin{aligned}
c_{4} & >\sqrt{2 c_{1} c_{2}}, \\
c_{1} c_{2} \log \varrho & >c_{1}+2 g(\varrho+1) c_{2} c_{4}, \\
c_{3} & =\max \left(\sqrt{c_{1}}, \sqrt{c_{2}}\right) .
\end{aligned}
$$

Note that $\gamma=R S /(K L)>2$ and then $g>1 / 4-1 / 24=5 / 24$. We easily check that there exist positive solutions of the system (4) which are as close as we wish to the limit values

$$
c_{1}=\frac{64 g^{2}(\varrho+1)^{2}}{(\log \varrho)^{3}}, \quad c_{2}=\frac{2}{\log \varrho}, \quad c_{3}=\max \left(\sqrt{c_{1}}, \sqrt{c_{2}}\right), \quad c_{4}=\sqrt{2 c_{1} c_{2}},
$$

with $g=5 / 24$. Strictly speaking, these values are not convenient because they lead to equalities in the first two relations of (4). Now choose $\varrho=5.8$, so that

$$
c_{1}=23.64 \ldots, \quad c_{2}=1.13 \ldots, \quad c_{3}=4.86 \ldots, \quad c_{4}=7.33 \ldots
$$

Theorem 3 yields the alternative: either

$$
\log \left|\Lambda^{\prime}\right| \geq-K L \log \varrho \geq-c_{1} c_{2} \log \varrho D^{4} B^{2} \log a_{1} \log a_{2},
$$

or there exist two integers $r$ and $s$, with $|r| \leq R-1,|s| \leq S-1$, such that $r b_{2}+s b_{1}=0$. Obviously we may suppose that $r$ and $s$ are relatively prime. Then from Lemma 2.2 in [4], we get

$$
|\Lambda| \geq\left|r \log \alpha_{1}+s \log \alpha_{2}\right| \geq \exp \left\{-D \log 2-D\left(|r| \log a_{1}+|s| \log a_{2}\right)\right\},
$$

which implies

$$
\log |\Lambda| \geq-D \log 2-2 c_{3} D^{5 / 2} B^{1 / 2} \log a_{1} \log a_{2}-2 c_{4} D^{3} B \log a_{1} \log a_{2} .
$$

Here, the main term is the third, which is better than required. To conclude, one has only to remark that for the above limit values, we have $c_{1} c_{2} \log \varrho<$ 48 and $\log \left|\Lambda^{\prime} / \Lambda\right|=\mathcal{O}(\log B)$.

For Theorem 2, the preceding arguments have to be made effective. We choose our constants slightly larger than the above limit values. Set

$$
\begin{gathered}
c_{1}=36, \quad c_{2}=1.5, \quad c_{3}=6, \quad c_{4}=6 \sqrt{3}+0.04=10.43 \ldots, \\
f=0.49, \quad \varrho=4.9 .
\end{gathered}
$$

Using systematically estimates of the type

$$
(x-0.04) y<[x y] \leq x y,
$$


which are true for any real numbers $x \geq 0$ and $y \geq 25$, we easily check that for $B \geq 25$, we have

$$
\begin{aligned}
R \leq 11.633 D^{2} B \log a_{2}, & S \leq 11.633 D^{2} B \log a_{1}, \\
\gamma \leq 2.578, \quad g & \leq 0.218
\end{aligned}
$$

(note that the third term from the definition of $g$ in Theorem 3 is bounded by $\left.10^{-4}\right)$. Lemma 9 gives the upper bounds

$$
b \leq \frac{5\left((R-1) b_{2}+(S-1) b_{1}\right)}{K-1} \leq \frac{5\left(R b_{2}+S b_{1}\right)}{K} \leq 1.62 b^{\prime}
$$

showing that $\log b \leq B$. Inequalities (1) are consequences of the lower bounds

$$
\begin{aligned}
R_{1} & \geq 6 D^{3 / 2} B^{1 / 2} \log a_{2}, & R_{2} & \geq 6 \sqrt{3} D^{2} B \log a_{2}, \\
S_{1} & \geq 6 D^{3 / 2} B^{1 / 2} \log a_{1}, & S_{2} & \geq 6 \sqrt{3} D^{2} B \log a_{1} .
\end{aligned}
$$

To check the main inequality (3), it is convenient to break it into two parts

$$
\begin{gathered}
K(L-1) \log \varrho>D(K-1) B+g(\varrho+1) D L\left(R \log a_{1}+S \log a_{2}\right), \\
(K-3) \log 2>2 D \log (K L) .
\end{gathered}
$$

The reader will easily check that the left hand side of $(3.1)$ is $\geq 81.15 D^{4} B^{2} \times$ $\log a_{1} \log a_{2}$, while the right hand side is $\leq 80.89 D^{4} B^{2} \log a_{1} \log a_{2}$, for $B \geq 25$. The condition (3.2) is quite surely satisfied for $B \geq 25$. By Theorem 3 , we know that either

$$
\log \left|\Lambda^{\prime}\right| \geq-K L \log \varrho \geq-86 D^{4} B^{2} \log a_{1} \log a_{2},
$$

or

$$
\log |\Lambda| \geq-D \log 2-D R \log a_{1}-D S \log a_{2} \geq-24 D^{3} B \log a_{1} \log a_{2} .
$$

Finally, the bound (quite weak for $B \geq 25$ )

$$
1+\log L+\log S+\log R \leq D^{4} B^{2} \log a_{1} \log a_{2}
$$

provides us with the required lower bound

$$
\log |\Lambda| \geq-87 D^{4} B^{2} \log a_{1} \log a_{2} .
$$

\section{References}

[1] J. Dieudonné, Calcul infinitésimal, Collection méthodes, Paris, 1968.

[2] M. Laurent, Sur quelques résultats récents de transcendance, Astérisque 198-200 (1991), 209-230.

[3] M. Mignotte and M. Waldschmidt, Linear forms in two logarithms and Schneider's method, Math. Ann. 231 (1978), 241-267.

[4] - - - Linear forms in two logarithms and Schneider's method II, Acta Arith. 53 (1989), 251-287. 
[5] M. Mignotte and M. Waldschmidt, Linear forms in two logarithms and Schneider's method III, Ann. Fac. Sci. Toulouse Math. 97 (1989), 43-75.

[6] M. Waldschmidt, Linear independence of logarithms of algebraic numbers, IMSc. Report No. 116, The Institute of Mathematical Sciences, Madras, 1992.

LABORATOIRE DE MATHÉMATIQUES DISCRÈTES

CNRS, UPR 9016

163 AVENUE DE LUMINY, CASE 930

13288 MARSEILLE CEDEX 9

FRANCE

Received on 11.6.1993

and in revised form on 22.9.1993 